\title{
High Energy Physics and Cosmology as Computation
}

\author{
Mohamed S. El Naschie \\ Department of Physics, Faculty of Science, University of Alexandria, Alexandria, Egypt \\ Email: Chaossf@aol.com
}

Received 25 June 2016; accepted 1 July 2016; published 4 July 2016

Copyright (C) 2016 by author and Scientific Research Publishing Inc.

This work is licensed under the Creative Commons Attribution International License (CC BY). http://creativecommons.org/licenses/by/4.0/

cC) (i) Open Access

\begin{abstract}
The present paper is basically written as a non-apologetic strong defence of the thesis that computation is part and parcel of a physical theory and by no means a mere numerical evaluation of the prediction of a theory which comes towards the end. Various general considerations as well as specific examples are given to illustrate and support our arguments. These examples range from the practical aspect to almost esoteric considerations but at the end, everything converges towards a unity of theory and computation presented in the form of modern fractal logic and transfinite quantum field theory in a Cantorian spacetime. It is true that all our examples are taken from physics but our discussion is applicable in equal measure to a much wider aspect of life.
\end{abstract}

\section{Keywords}

Fractal Logic, E-Infinity Theory, Cantorian-Fractal Spacetime, P. Erdos, A. Turing Computer, Transfinite Turing Machine, A. Connes Noncommutative Geometry, von Neumann Continuous Geometry, Golden Mean Computer, Pointless Geometry, Fuzzy Sets, Fuzzy Logic

\section{Introduction}

Mathematical computation aided by number theory [1]-[14], digital and analogue modern computers [14]-[27] are far more basic to quantum physics and cosmology than most theoretical physicists, including the present Author could usually have thought or imagined most of the time until relatively recently [22]-[39]. There are of course a few notable exceptions reaffirming the rule such as the remarkable French pure and applied mathematician Alain Connes [28] as well as the founding farther of so many branches of pure and applied mathematics, John von Neumann [29].

In the present work we take theoretical physics, exemplified by E-infinity fractal Cantorian theory [31]-[137] into the relatively new direction of science set in computational space to explore nature as a computation [3]-[5] 
[15]. This is actually the essence of E-infinity theory [31]-[137]. In turn and to close the circle, the core of E-infinity theory is a transfinite form of a Turing computer [14] [15]. Finally we will see immediately that the essence of this transfinite Turing machine is a simple golden mean based number system, which is at the heart of nature itself [3] [4] [11] [12].

Said in a few words, our present work is addressing scientists who love numbers but not only numbers such as P. Erdos and S. Ramanujan [19] [20]. To understand nature we need all the tools we can put our hands on and one cannot afford to dismiss computation as merely the trivial end result of a well understood theory. Computation gives us not only an insight into a theory but can also lead to a theory hiding in fine structure details which we did not even suspect that it existed before seeing the inner wheel of the computation process at work as we hope to show here [1]-[155].

\section{A Golden Mean Based Computation}

As is in the meantime well known, E-infinity theory makes extensive use of a golden mean based number system. Consequently we start by introducing two fundamental theorems [11] [12] [30].

Theorem 1

Any positive integer can be written uniquely as the sum of non-consecutive Fibonacci numbers of the following well known series:

$$
1,1,2,3,5,8,13,21,34,55, \cdots
$$

That means the Fibonacci series is given by:

$$
a_{n}=a_{n-1}+a_{n-2}
$$

Theorem 2

Any positive real number can be represented uniquely as the sum of non-consecutive number of the following series:

$$
\cdots, \phi^{3}, \phi^{2}, \phi, \frac{1}{\phi}, \frac{1}{\phi^{2}}, \frac{1}{\phi^{3}}, \cdots
$$

where $\phi=(\sqrt{5}-1) / 2$ is the golden mean which is the ratio of $a_{n-1} / a_{n}$ for $n \rightarrow \infty$. We discussed the theory and application of this number system as an alternative to traditional and conventional decimal and binary systems in many previous publications [31]-[36] [43] [44] [48] [52] [59] which the reader may consult intermittently as he reads the present paper.

\section{What We Ought to Have Learnt from Nonlinear Dynamics and Deterministic Chaos}

Deterministic chaos, a phrase coined by American mathematician and pioneer of nonlinear dynamics, chaos and fractal dynamics J. York [138]-[142], may seem to be a quite contradictory notion. However the message it conveys is very deep indeed and boils down to a peculiar fact, namely that most of what we take to be pure chance is in fact complex and purposeful and yet much of what we know to be a deterministic process is best tackled as if it were pure true randomness. In the light of such philosophy, number theory and some classical books such as that of Hardy [2] as well as the work of P. Erdos [17] [18] and S. Ramanujan [19] [20] became the new holy grail of nonlinear dynamics research [138]-[142]. In addition neither numerical experiments nor number theory were looked down upon, scorned or considered irrelevant to real physics in the relatively new and still vibrant field of deterministic chaos and fractals [137]-[145] [52]-[155]. The Author is rather of the mildly strong opinion that this is a healthy scientific attitude worth exporting from nonlinear dynamics to high energy quantum physics and cosmology [48] [141] and the next sections are concrete examples for what we mean by that.

\section{Deriving the Dark Energy and the Ordinary Energy Density of the Cosmos from the Classical Theory of $n$-Dimensional Spheres}

It is important in science to realize the difference between similar things without over estimating either. This is also a lesson from nonlinear dynamics and fractals. There we learnt the crucial difference between a topological 
dimension and a Hausdorff dimension. Never the less both are dimensions. The marvellous devise Hausdorff dimension [138] [141] on the other hand is closely related not only to the volume of an object but also to its irregularity and consequently it is related to its complexity, entropy [53] and therefore to its surface area, temperature and information. Now we could use this web of interrelationship to give a rather excellent estimation of the dark energy and ordinary energy density of spacetime of the cosmos. Naturally we could not proceed confidently in this way except for the fact that we solved the very same problem using a dozen or more exact methods so that we have considerable knowledge as well as feel and intuition for this problem that many regard as the most fundamental challenge facing modern cosmological research. That is maybe why we cannot hide the feeling of satisfaction and a sense of achievement to be able to give yet another extremely simple solution of this problem using well known and established classical geometry. To do that we need to recall the following facts [143][147].

a) The volume of a 3D, 4D and 5D ball is given by $4 \pi / 3, \pi^{2} / 2$ and $8 \pi^{2} / 15$ respectively.

b) The surface area of a five dimensional sphere is given by $8 \pi^{2} / 3$.

Now it is obvious that the $8 \pi^{2} / 3 \simeq 26$ of the five dimensional sphere is practically the empty set wave corresponding to the five dimensional cobordism of our four dimensional spacetime. Consequently $8 \pi^{2} / 3 \simeq 26$ may be regarded just as well as the Hausdorff dimension of the cobordism of our 4D spacetime and the five dimensionality as an enveloping Klein-Kaluza spacetime [148]-[150]. Adopting a slightly imaginative mathematical attitude towards the 3D volume of our 3D ball $4 \pi / 3 \simeq 4.18$, it can be regarded as the Hausdorff dimensionality of our spacetime which is quite close to its topological dimensionality $D=4$ and not far off its E-infinity Hausdorff dimensionality $D=4+\phi^{3} \simeq 4.236$. Similarly the volume for five dimensions, i.e. $8 \pi^{2} / 15$ may be seen as sufficiently close to the topological dimension of a K-K spacetime, namely five as well as the Hausdorff dimension of the fractal version of K-K [128] [150], namely $D=5+\phi^{3} \simeq 5.236$. The final result coming out of the above is far simpler than it seems. It is clear that the maximal value of dimensionless pure numbers describing the maximal possible energy must be the larger dimension, i.e. 26 minus the smallest dimension, i.e. 4. On the other hand the next largest possible must be 26 minus 5 . It follows then that the largest density under these circumstances must be in the projected ring between $D=5$ and $D=4$ and is given by the ratio [94] [110] [137]

$$
\gamma(D)=\frac{26-5}{26-4}=21 / 22
$$

Within an integer theory this is the exact value of the dark energy density of the cosmos. Consequently we can interpret the above as follows:

1) The 26 are the largest dimensions of space referred to in mainstream physics as a bosonic spacetime as for instance in the well known heterotic string theory of D. Gross et al.

2) The $26-4=22$ is clearly the number of compactified extra dimensions where dark energy and dark matter are hiding.

3) The $26-5=21$ where 25 is the lower critical dimension of the classical Nambu-Goto strings [135]-[137] and the associated Gupta-Bleuler quantization [135] [136] leading to the ghost-free condition $a=1, D=26$ and $a \leq 1, D \leq 25$ where a is the intercept.

Thus we demonstrated that the estimation of $\gamma(D)$ based on a classical theory, namely

$$
\gamma(D)=\frac{\left(8 \pi^{2} / 3\right)-5}{\left(8 \pi^{2} / 3\right)-4}=0.955195 \simeq 95.5 \%
$$

is accurate and far from being a coincidence. It is well founded and entails a unity of physics and geometry fused by computation. Needless to say, the above leads naturally to the conclusion that the ordinary energy density corresponding to $\gamma(D)=95.5 \%$ is $\gamma(O)=1-(95.0 \%)=4.5 \%$ and is given by the self explanatory equation [131]-[137]

$$
\gamma(O)=\frac{D^{(5)}-D^{(4)}}{D^{(26)}-D^{(4)}}=\frac{5-4}{26-4}=\frac{1}{22}=4.5 \%
$$

4) We could also estimate the dark energy density via the volume of a four and a five dimensional ball. It is quite easy to see that this may be done via the ratio of the two volumes and consequently we have

$$
\gamma(D)=\left(\pi^{2} / 2\right) /\left(8 \pi^{2} / 15\right)=15 / 16=0.9375
$$


which is not far off the exact value. To obtain the exact value we need to introduce transfinite "correction" as frequently done in E-infinity theory by noting that

$$
\pi^{2} / 2=4.9348 \rightarrow 5=D(K-K)
$$

and

$$
8 \pi^{2} / 15=5.2637 \rightarrow 5+\phi^{3}=D(K-K)
$$

The exact value is thus [131]-[137]

$$
\gamma(D)=\frac{5}{5+\phi^{3}}=5 \phi^{2} / 2=(21+k) /(22+k)
$$

In conclusion of this section we recall that the exact transfinite solution of the problem was based on a dissection of $E=m c^{2}$ of Einstein into two quantum components leading to

$$
E=\left(m c^{2} / 22+k\right)+m c^{2}(21+k / 22+k)
$$

where $k=\phi^{3}$ and $\phi=(\sqrt{5}-1) / 2$. In this dissection the first part is the energy of the quantum particle which is about $4.5 \%$ of total energy and since it can be measured it was found that it is nothing but the ordinary energy found by COBE and WMAP cosmological measurements [113]. By contrast the second term is the $95.5 \%$ missing energy of the cosmos and is due to the peculiarity of the quantum wave rather than the quantum particle. We will discuss this very same problem in connection with von Neumann-Connes dimensional function [28] [154] in the next section.

\section{From von Neumann-Connes Dimensional Function to Physics}

Physics-like mathematics is a subject that fascinated and occupied the present Author as a relatively young researcher during his time as a Visiting Professor at Cornell University and later on in Cambridge [21]-[24] [153]. However at that early point of time the Author was not aware of noncommutative geometry as developed and refined by A. Connes [28] building upon von Neumann's “pointless” continuous geometry [29] [154]. In the end it turned out that the work of A. Connes is closely related to a dimensional function describing the topology of a certain class of Penrose tiling-like manifolds. In turn this dimensional function $D=a+b \phi$ where $a, b \in Z$ and $\phi=(\sqrt{5}-1) / 2$ turned out to be completely equivalent to the bijection formula of the Author's E-infinity theory which states that [125] [154]

$$
d_{c}^{(n)}=\left(1 / d_{c}^{(o)}\right)^{n-1}
$$

where $n$ is a Menger-Urysohn deductive topological dimension, $d_{c}^{(n)}$ is the corresponding Hausdorff dimension and $d_{c}^{(o)}$ is the zero set [31]-[36].

Let us be explicit in deriving the most important two sets, namely the zero set modelling the pre-quantum particle and the empty set modelling the quantum wave and in the course of doing that lay bare the hidden identity of the dimensional function and the bijection formula. Because it is much easier to work with the bijection formula, we start from there. Setting $d_{c}^{(0)}=\phi$ and inserting in $d_{c}^{(n)}$ we see that for a Menger-Urysohn topological dimension $n=0$, i.e. the zero set we would have the Hausdorff dimension [31]-[36]

$$
d_{c}^{(n)}=d_{c}^{(o)}=\left(\frac{1}{\phi}\right)^{n-1}=\left(\frac{1}{\phi}\right)^{0-1}=\left(\frac{1}{\phi}\right)^{-1}=\phi
$$

exactly as should be. Next we look at the empty set defined by the inductive Menger-Urysohn topological dimension $n=-1$ and inserting again in the bijection formula we find

$$
d_{c}^{(n)}=d_{c}^{(-1)}=\left(\frac{1}{\phi}\right)^{-1-1}=\left(\frac{1}{\phi}\right)^{-2}=\phi^{2}
$$

Let us see how to reproduce the same result from the dimensional function. It is obvious that to obtain $D=\phi$, i.e. to obtain the zero set we must have $a=0$ and $b=1$ so that one finds the trivial result [28]-[38]

$$
D(0)=0+(1)+(\phi)=\phi=d_{c}^{(0)}
$$


To find the empty set on the other hand we must have $a=1$ and $b=-1$ from which one finds [28]-[38]

$$
D(-1)=1+(-1) \phi=1-\phi=\phi^{2}=d_{c}^{(-1)}
$$

Now the reader can see for himself that the topological Menger-Urysohn dimension is not immediately obvious from the structure of the dimensional function as it is trivially obvious from the equivalent formalism of the bijection formula. For instance to find $\mathrm{D}(\mathrm{O})$ we had to have $a=0$ but to find $D(-1)$ we had to have $b=-1$. Of course we could define the vital $a, b \in Z$ which corresponds to the topological MU dimension as (a) (b) so that for $\mathrm{D}(\mathrm{O})$ we find $D(T)=(0)(1)=0$ while for $D(-1)$ we find $D(T)=(1)(-1)=-1$ which is correct and consistent. However it is self evident that this way and although we arrive at the correct result is neither direct nor particularly illuminating. The alternative solution with a dimension function formalism is to stick to the Fibonacci-like recursive nature of the function as indicated at the very beginning. To do that we start best with the following seed $D(1,0)$ and $D(0,1)$ with which we mean [28]-[38]

$$
D(1,0)=1+0=1=D(1)
$$

and

$$
D(0,1)=1+\phi=D(2)
$$

Now by subtraction one finds

$$
D(0)=D(2)-D(1)=1+\phi-1=\phi=d_{c}^{(0)}
$$

and

$$
D(-1)=D(1)-D(0)=1-\phi=\phi^{2}=d_{c}^{(0)}
$$

We can go on in the same way and find

$$
D(-2)=D(0)-D(-1)=\phi-\phi^{2}=\phi^{3}
$$

and so on as explained in more details elsewhere. Notice that we could go into the "positive" dissection in an analogous way by adding instead of subtracting and find

$$
D(3)=D(2)+D(1)=1+\phi+1=2+\phi=\left(\frac{1}{\phi}\right)^{3-1}=\left(\frac{1}{\phi}\right)^{2}=d_{c}^{(2)}
$$

and

$$
D(4)=D(3)+D(2)=(2+\phi)+(1+\phi)=4+\phi^{3}=\left(\frac{1}{\phi}\right)^{4-1}=\left(\frac{1}{\phi}\right)^{3}=d_{c}^{(4)}
$$

and so on. In particular $D(4)=d_{c}^{(4)}$ is in the meantime the well known Hausdorff dimension of our quantumCantorian spacetime which cannot distinguish between union and intersection because of the unique number theoretical equality

$$
d_{c}^{(2)}+d_{c}^{(3)}=\left(d_{c}^{(2)}\right)\left(d_{c}^{(3)}\right)
$$

We can easily see that by inserting the relevant values in the above we find that

$$
\left(\frac{1}{\phi}\right)+\left(\frac{1}{\phi}\right)^{2}=\left(\frac{1}{\phi}\right)\left(\frac{1}{\phi}\right)^{3}=\left(\frac{1}{\phi}\right)^{3}=4+\phi^{3}=4.236067977 \text {. }
$$

Thus the expectation value of the dimensionality of spacetime is truly unique because

$$
D\left(4 ; 4+\phi^{3}\right)
$$

is indifferent to union and intersection with respect to both the topological dimension where $2+2=(2)(2)=4$ as well as the Hausdorff dimension because

$$
(2.618033988)+(1.618033989)=(2.618033988)(1.618033989)=4+\phi^{3}=4.23606797
$$


In this sense our micro-quantum spacetime is truly remarkable from a computational point of view and that is the reason for its "physical” uniqueness which prompts many scientists to ask why are we living in $3+1$ spacetime?

\section{Short Computational and Conceptual Contrast between Quantum Mechanics, String Theory and von Neumann-Connes-E-Infinity Fractal Strings}

It is clear that while E-infinity as well as noncommutative geometry are "space manifold" based quantum theories [28] [29] [31]-[33], orthodox quantum mechanics makes no reference to a spacetime manifold. Many computational consequences result from this fundamental difference [2]-[5]. We discuss this point very briefly and include superstring theory [151] for reasons that will shortly become apparent.

To start with in quantum mechanics we have quantum particles and quantum waves and hardly anything else. However there is no numeric assigned a priori to either a quantum particle or a quantum wave. In string theory we have some definite numbers because the vague point-like particles of quantum mechanics which have no spacetime. We have instead strings with a dimension equal that of a line, namely $\mathrm{D}=1$. The nearest we come to a fundamental thing resembling a wave in string theory would be the world sheet which has a dimension equal two so that we may start thinking of using two numbers, namely one and two. That way we may regard our 3D space as a string interacting with a world sheet while spacetime is the interaction of two world sheets. Other interpretations are of course possible and were proposed in different forms. This becomes more interesting as we move to E-infinity and von Neumann-Connes fractal strings [28]-[33]. Here we have besides the topological dimensions, a Hausdorff dimension. Thus the counterpart of a $D=1$ string is $D=1 / \phi=1+\phi$ which is the inverse of the Hausdorff dimension of the pre-quantum particle zero set which was replaced by a string in string theory. Numerically it is a string plus a pre-quantum particle giving rise to what we call a fractal string with a Hausdorff dimension equal $1+\phi=1.168033989$. Topologically it is however a two dimensional object because of the fact that $d_{c}^{(2)}=1 / \phi=1.168033989$. As for the fractal object corresponding to the $\mathrm{D}=2$ world sheet, we have in this case another object with $D=2+\phi=2.16803398$. This may be seen as a fractal string $1+\phi$ plus one non-fractal string interacting in union to give rise to $1+\phi+1=2+\phi$ or simply a pre-quantum particle in union with a classical string world sheet 2 giving rise to what we may call a fractal world sheet

$D=2+\phi=2.16803398$. This is clearly a three rather than two dimensional object because

$d_{c}^{(3)}=(1 / \phi)^{2}=2.16803398$. The interesting point is that adopting this fractal string picture we see the time dimension must be fractal in nature because a string plus a world sheet in classical string theory would lead to $1+$ 2 = 3 dimensional space but including the fractal fine structure the same sum would give

$$
(1+\phi)+(2+\phi)=(1+2)+2 \phi=4+\phi^{3}
$$

which means $d_{c}^{(4)}=4+\phi^{3}$ and this is our fractal spacetime dimensionality [134].

\section{Connectivity Dimension and the Electroweak Energy Scale}

The following derivation is yet another example of how mathematics and physics mingle via computation and result in the uncovering of unsuspected interrelations with deep insight and understanding. Let us start from a logarithmic "gauging" of the bijection formula [35] [36]

$$
d_{c}^{(n)}=\left(1 / d_{c}^{(0)}\right)^{n-1}
$$

That way one finds [69]

$$
\ln d_{c}^{(n)}=(n-1) \ln \left(1 / d_{c}^{(0)}\right) .
$$

Setting $n=D_{c}$ and $\left\langle 1 / d_{c}^{(0)}\right\rangle=2$ and solving for $D$ one finds

$$
D_{c}=\frac{\ln d_{c}^{(n)}}{\ln 2}+1 .
$$

Now $d_{c}^{(n)}$ could be taken to be a complexion of an intricate graph and $D_{c}$ then becomes the well known connectivity dimension of this complex. Setting as our complexion the largest physically known dimensionless number, then it is intuitively obvious that our best bet must be Newton's dimensionless gravity constant between 
two protons $G_{N} \simeq(1.7)\left(10^{38}\right)$ [69]. Inserting into our $D_{c}$ one finds a deep meaningful result, namely the inverse electromagnetic constant at the electroweak unification energy scale [69]

$$
D_{c}=\frac{\ln \left[(1.7)\left(10^{38}\right)\right]}{\ln 2}+1 \simeq 127+1 \simeq 128 \simeq \bar{\alpha}_{e w}
$$

Needless to say $\bar{\alpha}_{e w} \simeq 128$ is an experimentally well established result in no less measure than $\bar{\alpha}_{o} \simeq 137$ of electromagnetism [59] [69].

\section{Erdős Small World Connectivity and String Theory}

The legendary Hungarian number theoretician Paul Erdős [17] [18] came close to proving a remarkable theorem about connectivity which when translated to the language of modern social media would mean that it is sufficient to have only 24 acquaintances of each of us to connect almost the entire population of the planet earth. Based on this theorem the Author conjectured that the correct "dimensionality" for this aspect of small world social media must be $26+\mathrm{k}=26.18033989$ which is the bosonic strings fractal dimension [155]. Research in small world science runs in part in a way similar to nonlinear dynamics and chaos [155] and makes extensive use of numerical experiments with little regard for any undue reluctance to indulge in computational mathematics fearing the usual readymade labels, which we need not mention here. Rather than wasting time and space on opposing what is essentially human arrogance produced by ignorance we better discuss next a truly remarkable result of a man of the mold of Paul Erdős [17] [18] taken to the extreme. This man is S. Ramanujan [19] [20].

\section{From Fractal Logic and Cesaro Convergence to the Standard Model of High Energy Physics}

The following infinite sum must be one of the most absurd looking results because adding the positive integers from 1 to infinity in the history of mathematics could not in ordinary logic give a negative fraction. It was introduced by the Indian mathematical visionary S. Ramanujan [19] [20] and we can assure the reader that it is not only correct but also has various physical applications in physics including superstrings [151]

$$
\sum_{n=1}^{\infty} n=-\frac{1}{12}
$$

As usual in such situations the apparent absurdity is only apparent and lies in what we really mean with the mathematical symbols. The point is that the result $-1 / 12$ is the Cesaro limit of this otherwise naturally diverging series. We will not discuss the pure mathematics of this highly interesting field but will concentrate on its relation to our fractal logic as employed to counting the messenger particles of the standard model. We may recall that the standard model is based on $\mathrm{SU}(3), \mathrm{SU}(2)$ and $\mathrm{U}(1)$ Lie symmetry groups and that its constant dim $(\mathrm{SU}(3) \mathrm{SU}(2) \mathrm{U}(1))=12$ elementary messenger particles excluding the mass and gravity section which means that it is not complete [152]. Using our fractal logic counting we showed on many earlier occasions that the 12 particles have the weighted number 11.7082033 and means that we have 14 elementary messenger particles [152]. In other words we can write an equality which is not less absurd but in fact more outrageous in its appearance than the $-1 / 12$ sum of Ramanujan [20] introduced earlier on and states that:

$$
12=11.7082033925=14 \text {. }
$$

Now let us see if something useful could come out of this systematic madness which is never the less mathematically well founded and we expect that anything that is mathematically founded is logical and in turn it is our metaphysical conviction that it will be used by nature in one way or another. Rearranging one finds from the expression of the Cesaro limit of our series that [151]

$$
12=-\sum_{n=1}^{\infty} n=-1-2-3-4-\cdots
$$

Now all these negative integers could be seen in various different ways. Firs they could be seen as the MengerUrysohn dimensions with increasing degrees of emptiness. This means we are dealing with the true vacuum considered a multi-fractal of empty set with infinite hierarchal dimensions. Remembering that the expectation value of E-infinity Cantorian-fractal spacetime $D=4+\phi^{3}$ is the inversion of the dimensionality of the empty 
set surrounding the quantum wave, i.e. it is the empty set $\phi^{3}$ surrounding $\phi^{2}$, we see that the inverse of the vacuum multi-fractal empty set are the exact opposite dimension of finding the expectation value for spacetime such as $4+\phi^{3}$ or in fact Witten's fractal M-theory $11+\phi^{5}$ [128]. For instance if spacetime is taken as in Mtheory as $\mathrm{D}=11$, then setting a single photon in $D=11$ gives us a spacetime of the F-theory type with $11+1=12$ dimensions [128]. On the other hand in E-infinity theory as well as von Neumann-Connes theory the dimensionality 11 becomes $(1 / \phi)^{5}=11+\phi^{5}$ as in the fractal version of M-theory as the photon is given the weighted number $\phi$ rather than unity. Consequently $11+1=12$ becomes

$$
11+\phi^{5}+\phi=11.708203933=\sqrt{\bar{\alpha}_{o}}
$$

where $\bar{\alpha}_{o}=137.0820$ is the exact E-infinity inverse electromagnetic fine structure constant. In other words the fractal logic that is the true logic of counting in high energy physics and cosmology indicates that the fractal number of the elementary messenger bosons are indeed equal not 12 but 11.70820393 and that translated to our normal integer counting this number is 14 which means in turn that we can consider the standard model complete and include the graviton although it is overlooked theoretically for being essentially a fractal fine structure which makes it also even more difficult to detect experimentally than the illusive Higgs [152].

From the above admittedly complex discussion and somewhat subtle to the degree bordering on terse reasoning, we see that our Cesaro sum $-1 / 12$ is a relative of our theory and we could just interpret 12 as being $11+\phi^{5}+\phi=\sqrt{\bar{\alpha}_{o}}=11.7082033$ which is the fractal weight number of 14 elementary messenger particles. This is the reason why we can obtain the exact integer based value of the ordinary and the dark energy density of the cosmos from Witten's M-theory as [94] [128]

$$
E=\left(\frac{m c^{2}}{2}\right)\left[\frac{1}{11}+\frac{21}{11}\right]=\left(m c^{2} / 22\right)+m c^{2}(21 / 22)=m c^{2}
$$

while the exact transfinite expression is given by Witten’s fractal M-theory as [94] [128]

$$
\begin{aligned}
E & =\frac{m c^{2}}{2}\left[\frac{1}{11+\phi^{5}}+\frac{21+k}{11+\phi^{5}}\right]=m c^{2}\left(\phi^{5} / 2\right)+m c^{2}\left(5 \phi^{2} / 2\right) \\
& =\left(m c^{2} / 2\right)\left(\phi^{5}+5 \phi^{2}\right)=m c^{2}=E(\text { Einstein })
\end{aligned}
$$

In conclusion of this section we may mention on passing a remarkable decomposition of $E=m c^{2}$ into three parts corresponding and agreeing with the actual cosmic measurements of ordinary energy, dark matter and pure dark energy of the cosmos

$$
\begin{aligned}
E & =E(O)+E(D M)+E(D E) \\
& =m c^{2}\left[\frac{1}{22+k}+\frac{5-k_{o}}{22+k}+\frac{16+k+k_{o}}{22+k}\right]
\end{aligned}
$$

where $k=\phi^{3}\left(1-\phi^{3}\right)$ is 'tHooft's renormalon and $k_{o}=\phi^{5}\left(1-\phi^{5}\right)$ with $\phi^{5}$ being Hardy's entangleon.

\section{Discussion}

When the great E. Witten set out to construct his acclaimed M-theory he rightly chose to start with the most important invariant of a topology and reasoned that he needed seven spacetime dimensions to embed the Lie symmetry groups of the standard model, i.e. the $\mathrm{SU}(3) \mathrm{SU}(2) \mathrm{U}(1)$. Consequently an optimal spacetime must be the sum of Einstein's 4 spacetime dimensions plus these seven and so he arrived at the same dimension of super gravity, namely [128]

$$
D_{M}=D^{(4)}+D^{(7)}=4+7=11
$$

Now the Author asks the reader to pay special attention to how our transfinite computational methodology leads almost automatically to the same result and even a little more. First we know that our quantum spacetime most important dimensionality is that of its core, namely $4+\phi^{3}$ which is nothing more than $(1 / \phi)^{3}=4.236067977$. Next we ask what is the dimensionality of the space that constitutes the boundary of this core? Clearly by an obvious cobordism argument, the boundary space must be given by $(1 / \phi)^{4}=6.854201959$. Together core space 
and boundary space amount to the union, i.e. the sum of both dimensions. This means

$$
D(\text { total })=(1 / \phi)^{3}+(1 / \phi)^{4}=4+\phi^{3}+7-\phi^{4}=(4+7)+\left(\phi^{3}-\phi^{4}\right)
$$

This is a remarkable way to reproduce Witten's famous result, since $\phi^{3}-\phi^{4}$ is a very small value compared to the $4+7=11$. Thus neglected the small irrational tail we find in the original dimensionality of M-theory

$$
D_{M}=4+7=11
$$

However if we insist upon being pedantically accurate and include the small $\phi^{3}-\phi^{4}=\phi^{5}$ we are rewarded with a pleasant surprising result, namely that $\phi^{5}$ is Hardy's celebrated probability of quantum entanglement of two quantum particles. In addition the continued fraction of the exact value becomes

$$
D_{M F}^{(11)}=4+7+\phi^{3}-\phi^{4}=11+\phi^{4}=11+\frac{1}{11+\frac{1}{11+\cdots}}
$$

Thus we find a Russian doll-like 11-D Witten spacetime inside another 11-D spacetime and so on indefinitely. The reader may also be intrigued to see that the dimensionality of enveloping space given by $(1 / \phi)^{4}$ is simply $(1 / \phi)^{5}=11+\phi^{5}$ and is nothing more than the inverse of Hardy's quantum entanglement $\phi^{5}$. This is the beginning of discovering the fractal version of Witten's M-theory and at the same time, the main message of the present paper. At the risk of being a little repetitive, computation is an indispensible part of the theory and not only mere numeric. That is why we see meaning and physics in results that at first glance may seem absurd and faulty although it is not such as $\sum_{1}^{\infty} n=-1 / 12$ of Ramanujan [19] [20] and the many other results such as our equation No. 31 reported in the present paper.

\section{Conclusion}

Computation lies at the root of the very process of thinking. It is not the last stage of a theory but more often than not, it should be at the beginning of theoreticizing. Number systems can help us present a theory in a much simpler way than thought possible and the present Author suspects that nature is basically a kind of gigantic computer of the transfinite golden mean based Turing type. What distinguishes this computer from the classical Turing machine is the software which we allege is based on a transfinite golden mean binary system introduced at the very beginning of the present paper and is therefore virtually infinitely more powerful than any known computer. There are many implicit results and consequences in the present work which we did not mention due to space limitation, for instance the mere fact that $\phi+\phi^{2}=1$ shows where the roots of the problem of fine tuning in physics and cosmology lies. We hope to come to these and similar points in the future.

\section{Acknowledgements}

This paper is indebted to the work of A. Connes, G. Hardy and S. Ramanujan. It is dedicated to peace in the Middle East and the ideal of a United States of The Middle East, which is a natural demand arising from applying computational fractal logic to a most troubled part of our world.

\section{References}

[1] Waldschmidt, M., Moussa, P., Luck, J. and Itzykson, C. (1992) From Number Theory to Physics. Springer-Verlag, Berlin. http://dx.doi.org/10.1007/978-3-662-02838-4

[2] Hardy, G.H. and Wright, E.M. (1979) An Introduction to the Theory of Numbers. Clarendon Press, Oxford, UK.

[3] Zenil, H. (Editor) (2013) A Computable Universe. World Scientific, Singapore.

[4] Tegmark, M. (2014) Our Mathematical Universe. Allen Lane-Penguin Books, London.

[5] Lloyd, S. (2005) Programming the Universe. Jonathan Cape, London.

[6] Pickover, C.A. (1990) Computers, Patterns Chaos and Beauty. St. Martin’s Press, New York.

[7] Beiler, A.H. (1966) Recreations in the Theory of Numbers. Dover Publications, New York.

[8] Dedekind, R. (1963) Essays on the Theory of Numbers. Dover Publications, New York. 
[9] Artman, B. (1988) The Concept of Numbers. Ellis Horwood Ltd.-John Wiley, New York.

[10] Deutsch, D. (2011) The Beginning of Infinity. Allen Lane-Penguin Books, London.

[11] Posamentier, A.S. and Lehmann, I. (2007) The Fabulous Fibonacci Numbers. Prometheus Books, New York.

[12] Stakhov, A. (2009) The Mathematics of Harmony. World Scientific, Singapore.

[13] Changeux, J.-P. and Connes, A. (1989) Conversations on Mind, Matter and Mathematics. Princeton University Press, Princeton.

[14] Rendell, P. (2016) Turing Machine in Conway Game of Life. In: Designing Beauty: The Art of Cellular Automata, Springer, Heidelberg, 149-154. http://dx.doi.org/10.1007/978-3-319-27270-2_25

[15] Deutsch, D. (1985) Quantum Theory, the Church-Turing Principle and the Universal Computer. Proceedings of the Royal Society A, Mathematical, Physical and Engineering Sciences, 400, 97-117.

[16] Turing, A.M. (1950) Computing Machinery and Intelligence. Mind, 59, 433-460. http://dx.doi.org/10.1093/mind/LIX.236.433

[17] Babai, L., Pomerance, C. and Vertesi, P. (1998) The Mathematics of Paul Erdos. Notices of the American Mathematical Society, 45, 19-31.

[18] Hoffman, P. (1998) The Man Who Loved Only Numbers. Hyperion, New York.

[19] Kanigel, R. (1991) The Man Who Knew Infinity. Scribners, New York.

[20] Ramanujan, S. (2015) Collected Papers of Ramanujan. Cambridge University Press, Cambridge.

[21] El Naschie, M.S. (1991) Physics-Like Mathematical Model for Chaos and Ergodic Criticality in Four Dimensions. 13th Canadian Congress of Applied Mechanics (CANCAM 91), Paper No. A90.19, 756-757.

[22] El Naschie, M.S. (1991) Mathematical Model for Chaos and Ergodic Criticality in Four Dimensions. Mathematical and Computer Modelling, 15, 77-80. http://dx.doi.org/10.1016/0895-7177(91)90042-6

[23] El Naschie, M.S. (1992) Complex Dynamics in a 4D Peano-Hilbert Space. Il Nuovo Cimento, 107B, 583-594. http://dx.doi.org/10.1007/BF02723635

[24] El Naschie, M.S. (1992) Multi-Dimensional Cantor-Like Sets and Ergodic Behaviour. Speculations in Science \& Technology, 15, 138-142.

[25] El Naschie, M.S. (1993) On Universal Behaviour and Statistical Mechanics of Multi-Dimensional Triadic Cantor Sets. Systems Analysis Modelling Simulation, 11, 217-225.

[26] El Naschie, M.S. (1993) On Turbulence and Complex Dynamics in a Four Dimensional Peano-Hilbert Space. Journal of the Franklin Institute, 330, 183-198. http://dx.doi.org/10.1016/0016-0032(93)90029-T

[27] El Naschie, M.S. (1995) Statistical Geometry of a Cantor Discretum and Semi Conductors. Computers \& Mathematics with Applications, 29, 103-110. http://dx.doi.org/10.1016/0898-1221(95)00062-4

[28] Connes, A. (1994) Noncommutative Geometry. Academic Press, San Diego.

[29] Von Neumann, J. (1960) Continuous Geometry. Vol. 25, Princeton University Press, Princeton.

[30] Kappraff, J. (1991) Connections. McGraw-Hill Inc., New York.

[31] El Naschie, M.S. (2004) A Review of E-Infinity Theory and the Mass Spectrum of High Energy Particle Physics. Chaos, Solitons \& Fractals, 19, 209-236. http://dx.doi.org/10.1016/S0960-0779(03)00278-9

[32] El Naschie, M.S. (2005) A Guide to the Mathematics of E-Infinity Cantorian Spacetime Theory. Chaos, Solitons \& Fractals, 25, 955-964. http://dx.doi.org/10.1016/j.chaos.2004.12.033

[33] El Naschie, M.S. (2004) The Concepts of E-Infinity: An Elementary Introduction to the Cantorian-Fractal Theory of Quantum Physics. Chaos, Solitons \& Fractals, 22, 495-511. http://dx.doi.org/10.1016/j.chaos.2004.02.028

[34] El Naschie, M.S. (2006) Elementary Prerequisites for E-Infinity (Recommended Background Readings in Nonlinear Dynamics, Geometry and Topology). Chaos, Solitons \& Fractals, 30, 579-605. http://dx.doi.org/10.1016/j.chaos.2006.03.030

[35] El Naschie, M.S. (2007) A Review of Application and Results of E-Infinity. International Journal of Nonlinear Science \& Numerical Simulation, 8, 11-20. http://dx.doi.org/10.1515/IJNSNS.2007.8.1.11

[36] El Naschie, M.S. (2009) The Theory of Cantorian Spacetime and High Energy Particle Physics (An Informal Review). Chaos, Solitons \& Fractals, 41, 2635-2646. http://dx.doi.org/10.1016/j.chaos.2008.09.059

[37] El Naschie, M.S. (2006) On an Eleven Dimensional E-Infinity Fractal Spacetime Theory. International Journal of Nonlinear Sciences and Numerical Simulation, 7, 407-409.

[38] El Naschie, M.S. (2011) Quantum Entanglement as a Consequence of a Cantorian Micro Spacetime Geometry. Journal of Quantum Information Science, 1, 50-53. http://dx.doi.org/10.4236/jqis.2011.12007 
[39] El Naschie, M.S. (2007) Feigenbaum Scenario for Turbulence and Cantorian E-Infinity Theory of High Energy Particle Physics. Chaos, Solitons \& Fractals, 32, 911-915. http://dx.doi.org/10.1016/j.chaos.2006.08.014

[40] El Naschie, M.S. (2005) On a Fuzzy Kähler-Like Manifold Which Is Consistent with the Two Slit Experiment. International Journal of Nonlinear Sciences and Numerical Simulation, 6, 95-98. http://dx.doi.org/10.1515/IJNSNS.2005.6.2.95

[41] El Naschie, M.S. (2007) On the Topological Ground State of E-Infinity Spacetime and the Super String Connection. Chaos, Solitons \& Fractals, 32, 468-470. http://dx.doi.org/10.1016/j.chaos.2006.08.011

[42] El Naschie, M.S. (2005) On the Cohomology and Instantons Number in E-Infinity Cantorian Spacetime. Chaos, Solitons \& Fractals, 26, 13-17. http://dx.doi.org/10.1016/j.chaos.2004.12.019

[43] El Naschie, M.S. (2006) Advanced Prerequisites for E-Infinity Theory. Chaos, Solitons \& Fractals, 30, 636-641. http://dx.doi.org/10.1016/j.chaos.2006.04.044

[44] El Naschie, M.S. (2006) Topics in the Mathematical Physics of E-Infinity Theory. Chaos, Solitons \& Fractals, 30 , 656-663. http://dx.doi.org/10.1016/j.chaos.2006.04.043

[45] El Naschie, M.S. (2005) The Two-Slit Experiment as the Foundation of E-Infinity of High Energy Physics. Chaos, Solitons \& Fractals, 25, 509-514. http://dx.doi.org/10.1016/j.chaos.2005.02.016

[46] El Naschie, M.S. (2006) Holographic Dimensional Reduction: Center Manifold Theorem and E-Infinity. Chaos, Solitons \& Fractals, 29, 816-822. http://dx.doi.org/10.1016/j.chaos.2006.01.013

[47] El Naschie, M.S. (2006) Fuzzy Dodecahedron Topology and E-Infinity Spacetime as a Model for Quantum Physics. Chaos, Solitons \& Fractals, 30, 1025-1033. http://dx.doi.org/10.1016/j.chaos.2006.05.088

[48] El Naschie, M.S. (2006) Elementary Number Theory in Superstring Loop Quantum Mechanics, Twistors and E-Infinity High Energy Physics. Chaos, Solitons \& Fractals, 27, 297-330. http://dx.doi.org/10.1016/j.chaos.2005.04.116

[49] El Naschie, M.S. (2006) Hilbert, Fock and Cantorian Spaces in the Quantum Two-Slit Gedanken Experiment. Chaos, Solitons \& Fractals, 27, 39-42. http://dx.doi.org/10.1016/j.chaos.2005.04.094

[50] El Naschie, M.S. (2005) From Experimental Quantum Optics to Quantum Gravity via a Fuzzy Kähler Manifold. Chaos, Solitons \& Fractals, 25, 969-977. http://dx.doi.org/10.1016/j.chaos.2005.02.028

[51] El Naschie, M.S. (2008) Symmetry Group Prerequisite for E-Infinity in High Energy Physics. Chaos, Solitons \& Fractals, 35, 202-211. http://dx.doi.org/10.1016/j.chaos.2007.05.006

[52] El Naschie, M.S. (2006) Intermediate Prerequisites for E-Infinity Theory. Chaos, Solitons \& Fractals, 30, 622-628. http://dx.doi.org/10.1016/j.chaos.2006.04.042

[53] El Naschie, M.S. (2006) Superstrings, Entropy and the Elementary Particles Content of the Standard Model. Chaos, Solitons \& Fractals, 29, 48-54. http://dx.doi.org/10.1016/j.chaos.2005.11.032

[54] El Naschie, M.S. (2006) Hilbert Space, the Number of Higgs Particles and the Quantum Two-Slip Experiment. Chaos, Solitons \& Fractals, 28, 9-13. http://dx.doi.org/10.1016/j.chaos.2005.05.010

[55] El Naschie, M.S. (2006) On Two New Fuzzy Kähler Manifolds, Klein Modular Space and ‘tHooft’s Holographic Principles. Chaos, Solitons \& Fractals, 29, 876-881. http://dx.doi.org/10.1016/j.chaos.2005.12.027

[56] El Naschie, M.S. (2006) The Idealized Quantum Two-Slit Gedanken Experiment Revisited—Criticism and Reinterpretation. Chaos, Solitons \& Fractals, 27, 843-849. http://dx.doi.org/10.1016/j.chaos.2005.06.002

[57] El Naschie, M.S. (2003) Modular Groups in Cantorian E-Infinity High Energy Physics. Chaos, Solitons \& Fractals, 16, 353-366. http://dx.doi.org/10.1016/S0960-0779(02)00440-X

[58] El Naschie, M.S. (2003) E (Infinity) Cantorian Space-Time and Its Consequences in Cosmology. Chaos, Solitons \& Fractals, 25, 775-779.

[59] Helal, M., Marek-Crnjac, L. and He, J.-H. (2013) The Three Page Guide to the Most Important Results of M.S. El Naschie’s Research in E-Infinity Quantum Physics. Open Journal of Microphysics, 3, 141-145. http://dx.doi.org/10.4236/ojm.2013.34020

[60] El Naschie, M.S. (2006) The Brain and E-Infinity. International Journal of Nonlinear Science \& Numerical Simulation, 7, 129-132. http://dx.doi.org/10.1515/IJNSNS.2006.7.2.129

[61] El Naschie, M.S. (2006) E-Infinity-Some Recent Results and New Interpretations. Chaos, Solitons \& Fractals, 29, 845-853. http://dx.doi.org/10.1016/j.chaos.2006.01.073

[62] El Naschie, M.S. (2004) The Symplictic Vacuum, Exotic Quasi Particles and Gravitational Instanton. Chaos, Solitons \& Fractals, 22, 1-11. http://dx.doi.org/10.1016/j.chaos.2004.01.015

[63] El Naschie, M.S. (2003) The VAK of Vacuum Fluctuation, Spontaneous Self Organization and Complexity Theory Interpretation of High Energy Particle Physics and the Mass Spectrum. Chaos, Solitons \& Fractals, 18, 579-605. 
[64] El Naschie, M.S. (2008) Quasi Exceptional E12 Lie Symmetry Group with 685 Dimensions, KAC-Moody Algebra and E-Infinity Cantorian Spacetime. Chaos, Solitons \& Fractals, 38, 990-992. http://dx.doi.org/10.1016/j.chaos.2008.06.015

[65] Iovane, G. and Benedetto, E. (2006) El Naschie E-Infinity Cantorian Spacetime and Length Scales in Cosmology. International Journal of Nonlinear Sciences and Numerical Simulation, 7, 155-162. http://dx.doi.org/10.1515/ijnsns.2006.7.2.155

[66] He, J.-H. (2006) Application of E-Infinity Theory to Biology. Chaos, Solitons \& Fractals, 28, 285-289. http://dx.doi.org/10.1016/j.chaos.2005.08.001

[67] El Naschie, M.S. (2003) Non-Linear Dynamics and Infinite Dimensional Topology in High Energy Particle Physics. Chaos, Solitons \& Fractals, 17, 2635-2646. http://dx.doi.org/10.1016/S0960-0779(02)00402-2

[68] El Naschie, M.S. (2007) Deterministic Quantum Mechanics versus Classical Mechanical Indeterminism. International Journal of Nonlinear Science \& Numerical Simulation, 8, 5-10. http://dx.doi.org/10.1515/IJNSNS.2007.8.1.5

[69] El Naschie, M.S. (2004) Quantum Gravity, Clifford Algebras, Fuzzy Set Theory and the Fundamental Constants of Nature. Chaos, Solitons \& Fractals, 20, 437-450. http://dx.doi.org/10.1016/j.chaos.2003.09.029

[70] El Naschie, M.S. (2005) On Penrose View of Transfinite Sets and Computability and the Fractal Character of E-Infinity Spacetime. Chaos, Solitons \& Fractals, 25, 531-533. http://dx.doi.org/10.1016/j.chaos.2005.01.001

[71] Iovane, G. and Giordano, P. (2007) Wavelets and Multiresolution Analysis: Nature of E-Infinity Cantorian Spacetime. Chaos, Solitons \& Fractals, 32, 896-910. http://dx.doi.org/10.1016/j.chaos.2005.11.097

[72] Sigalotti, L.D.G. and Mejias, A. (2006) On El Naschie’s Conjugate Complex, Time, Fractal E-Infinity Spacetime and Faster than Light. International Journal of Nonlinear Science \& Numerical Simulation, 7, 467-472. http://dx.doi.org/10.1515/IJNSNS.2006.7.4.467

[73] El Naschie, M.S. (2003) Kleinian Groups in E-Infinity and Their Connection to Particle Physics and Cosmology. Chaos, Solitons \& Fractals, 16, 637-649. http://dx.doi.org/10.1016/S0960-0779(02)00489-7

[74] El Naschie, M.S. (2006) Is Gravity Less Fundamental than Elementary Particles Theory? Critical Remarks on Holography and E-Infinity Theory. Chaos, Solitons \& Fractals, 29, 803-807. http://dx.doi.org/10.1016/j.chaos.2006.01.012

[75] El Naschie, M.S. (2013) A Unified Newtonian-Relativistic Quantum Resolution of Supposedly Missing Dark Energy of the Cosmos and the Constancy of the Speed of Light. International Journal of Modern Nonlinear Theory \& Application, 2, 43-54. http://dx.doi.org/10.4236/ijmnta.2013.21005

[76] El Naschie, M.S. (2005) On a Class of Fuzzy Kähler-Like Manifolds. Chaos, Solitons \& Fractals, 26, 477-481. http://dx.doi.org/10.1016/j.chaos.2004.12.024

[77] El Naschie, M.S. (2006) A Cold Fusion-Casimir Energy Nano Reactor Proposal. World Journal of Nano Science and Engineering, 5, 49-56. http://dx.doi.org/10.4236/wjnse.2015.52007

[78] El Naschie, M.S. (2006) Fractal Black Holes and Information. Chaos, Solitons \& Fractals, 29, 23-35. http://dx.doi.org/10.1016/j.chaos.2005.11.079

[79] El Naschie, M.S. (2005) A Few Hints and Some Theorems about Witten’s M-Theory and T-Duality. Chaos, Solitons \& Fractals, 25, 545-548. http://dx.doi.org/10.1016/j.chaos.2005.01.009

[80] Marek-Crnjac, L. and He, J.-H. (2013) An Invitation to El Naschie’s Theory of Cantorian Spacetime and Dark Energy. International Journal of Astronomy and Astrophysics, 3, 464-471. http://dx.doi.org/10.4236/ijaa.2013.34053

[81] Iovane, G. (2006) El Naschie E-Infinity Cantorian Spacetime and Length Scales in Cosmology. International Journal of Nonlinear Sciences and Numerical Simulation, 7, 155-162. http://dx.doi.org/10.1515/ijnsns.2006.7.2.155

[82] El Naschie, M.S. (2007) The Fibonacci Code behind Superstrings and P-Branes. An Answer to M. Kaku's Fundamental Question. Chaos, Solitons \& Fractals, 31, 537-547. http://dx.doi.org/10.1016/j.chaos.2006.07.001

[83] Marek-Crnjac, L., El Naschie, M.S. and He, J.-H. (2013) Chaotic Fractals at the Root of Relativistic Quantum Physics and Cosmology. International Journal of Modern Nonlinear Theory and Application, 2, 78-88. http://dx.doi.org/10.4236/ijmnta.2013.21a010

[84] El Naschie, M.S. (2007) Towards a Quantum Golden Field Theory. International Journal of Nonlinear Sciences and Numerical Simulation, 8, 477-482. http://dx.doi.org/10.1515/IJNSNS.2007.8.4.477

[85] El Naschie, M.S. (2007) The Cosmic Da Vinci Code for the Big Bang-A Mathematical Toy Model. International Journal of Nonlinear Sciences and Numerical Simulation, 8, 191-194. http://dx.doi.org/10.1515/IJNSNS.2007.8.2.191

[86] El Naschie, M.S. (1998) Superstrings, Knots and Noncommutative Geometry in E-Infinity Space. International Journal of Theoretical Physics, 37, 2935-2951. http://dx.doi.org/10.1023/A:1026679628582

[87] El Naschie, M.S. (2004) Transfinite Electrical Networks, Spinoral Varieties and Gravity Q Bits. International Journal of Nonlinear Sciences and Numerical Simulation, 5, 191-198. 
[88] Iovane, G., Laserra, E., El Naschie, M.S. and Tortoriello, F.S. (2004) Stochastic Self Similar and Fractal Universe. Chaos, Solitons \& Fractals, 3, 415-426. http://dx.doi.org/10.1016/j.chaos.2003.08.004

[89] El Naschie, M.S. (2007) On the Universality Class of All Universality Classes and E-Infinity Spacetime Physics. Chaos, Solitons \& Fractals, 32, 927-936. http://dx.doi.org/10.1016/j.chaos.2006.08.017

[90] He, J.-H., Liu, Y., Xu, L. and Yu, J.Y. (2007) Micro Sphere with Nanoporosity by Electrospinning. Chaos, Solitons \& Fractals, 32, 1096-1100. http://dx.doi.org/10.1016/j.chaos.2006.07.045

[91] El Naschie, M.S. (2006) Superstring Theory: What It Cannot Do but E-Infinity Could. Chaos, Solitons \& Fractals, 29, 65-68. http://dx.doi.org/10.1016/j.chaos.2005.11.021

[92] El Naschie, M.S. (2014) Cosmic Dark Energy from ‘t Hooft’s Dimensional Regularization and Witten’s Topological Quantum Field Pure Gravity. Journal of Quantum Information Science, 4, 83-91. http://dx.doi.org/10.4236/jqis.2014.42008

[93] El Naschie, M.S. (2015) Kerr Black Hole Geometry Leading to Dark Matter and Dark Energy via E-Infinity Theory and the Possibility of Nano Spacetime Singularity Reactor. Natural Science, 7, 210-225. http://dx.doi.org/10.4236/ns.2015.74024

[94] El Naschie, M.S. (2014) From $E=m c^{2}$ to $E=m c^{2} / 22-$ A Short Account of the Most Famous Equation in Physics and Its Hidden Quantum Entangled Origin. Journal of Quantum Information Science, 4, 284-291. http://dx.doi.org/10.4236/jqis.2014.44023

[95] El Naschie, M.S. (2014) Pinched Material Einstein Space-Time Produces Accelerated Cosmic Expansion. International Journal of Astronomy and Astrophysics, 4, 80-90. http://dx.doi.org/10.4236/ijaa.2014.41009

[96] El Naschie, M.S. (2014) On a New Elementary Particle from the Disintegration of the Symplectic 't Hooft-VeltmanWilson Fractal Spacetime. World Journal of Nuclear Science and Technology, 4, 216-221. http://dx.doi.org/10.4236/wjnst.2014.44027

[97] El Naschie, M.S. (2014) The Measure Concentration of Convex Geometry in a Quasi Banach Spacetime behind the Supposedly Missing Dark Energy of the Cosmos. American Journal of Astronomy \& Astrophysics, 2, 72-77. http://dx.doi.org/10.11648/j.ajaa.20140206.13

[98] He, J.-H. (2014) A Tutorial Review on Fractal Spacetime and Fractional Calculus. International Journal of Theoretical Physics, 53, 3698-3718. http://dx.doi.org/10.1007/s10773-014-2123-8

[99] El Naschie, M.S. (2014) Electromagnetic-Pure Gravity Connection via Hardy’s Quantum Entanglement. Journal of Electromagnetic Analysis and Applications, 6, 233-237. http://dx.doi.org/10.4236/jemaa.2014.69023

[100] El Naschie, M.S. (2015) An Exact Mathematical Picture of Quantum Spacetime. Advances in Pure Mathematics, 5, 560-570. http://dx.doi.org/10.4236/apm.2015.59052

[101] El Naschie, M.S. (2015) A Resolution of the Black Hole Information Paradox via Transfinite Set Theory. World Journal of Condensed Matter Physics, 5, 249-260. http://dx.doi.org/10.4236/wjcmp.2015.54026

[102] Tang, W., Li, Y., Kong, H.Y. and El Naschie, M.S. (2014) From Nonlocal Elasticity to Nonlocal Spacetime and Nanoscience. Bubbfil Nano Technology, 1, 3-12.

[103] El Naschie, M.S. (2014) To Dark Energy Theory from a Cosserat-Like Model of Spacetime. Problems of Nonlinear Analysis in Engineering Systems, 20, 79-98.

[104] El Naschie, M.S. (2015) The Cantorian Monadic Plasma behind the Zero Point Vacuum Spacetime Energy. American Journal of Nano Research \& Application, 3, 66-70.

[105] El Naschie, M.S. (2015) The Casimir Topological Effect and a Proposal for a Casimir-Dark Energy Nano Reactor. World Journal of Nano Science \& Engineering, 5, 26-33. http://dx.doi.org/10.4236/wjnse.2015.51004

[106] El Naschie, M.S. (2014) Calculating the Exact Experimental Density of the Dark Energy in the Cosmos Assuming a Fractal Speed of Light. International Journal of Modern Nonlinear Theory and Application, 3, 1-5. http://dx.doi.org/10.4236/ijmnta.2014.31001

[107] El Naschie, M.S., Marek-Crnjac, L., Helal, M.A. and He, J.-H. (2014) A Topological Magueijo-Smolin Varying Speed of Light Theory, the Accelerated Cosmic Expansion and the Dark Energy of Pure Gravity. Applied Mathematics, 5, 1780-1790. http://dx.doi.org/10.4236/am.2014.512171

[108] El Naschie, M.S. (2015) On a Non-Perturbative Quantum Relativity Theory Leading to a Casimir-Dark Energy Nanotech Reactor Proposal. Open Journal of Applied Sciences, 5, 313-324. http://dx.doi.org/10.4236/ojapps.2015.57032

[109] El Naschie, M.S. (2014) From Chern-Simon, Holography and Scale Relativity to Dark Energy. Journal of Applied Mathematics and Physics, 2, 634-638. http://dx.doi.org/10.4236/jamp.2014.27069

[110] El Naschie, M.S. (2014) Why E Is Not Equal to $m c^{2}$. Journal of Modern Physics, 5, 743-750. http://dx.doi.org/10.4236/jmp.2014.59084 
[111] El Naschie, M.S. (2014) Capillary Surface Energy Elucidation of the Cosmic Dark Energy—Ordinary Energy Duality. Open Journal of Fluid Dynamics, 4, 15-17. http://dx.doi.org/10.4236/ojfd.2014.41002

[112] El Naschie, M.S. (2014) Cosmic Dark Energy Density from Classical Mechanics and Seemingly Redundant Riemannian Finitely Many Tensor Components of Einstein’s General Relativity. World Journal of Mechanics, 4, 153-156. http://dx.doi.org/10.4236/wjm.2014.46017

[113] El Naschie, M.S. (2015) Dark Energy and Its Cosmic Density from Einstein’s Relativity and Gauge Fields Renormalization Leading to the Possibility of a New 'tHooft Quasi Particle. The Open Astronomy Journal, 8, 1-17. http://dx.doi.org/10.2174/1874381101508010001

[114] El Naschie, M.S. (2015) If Quantum “Wave” of the Universe Then Quantum "Particle” of the Universe: A Resolution of the Dark Energy Question and the Black Hole Information Paradox. International Journal of Astronomy \& Astrophysics, 5, 243-247. http://dx.doi.org/10.4236/ijaa.2015.54027

[115] El Naschie, M.S. (2014) From Modified Newtonian Gravity to Dark Energy via Quantum Entanglement. Journal of Applied Mathematics and Physics, 2, 803-806. http://dx.doi.org/10.4236/jamp.2014.28088

[116] El Naschie, M.S. (2014) From Highly Structured E-Infinity Rings and Transfinite Maximally Symmetric Manifolds to the Dark Energy Density of the Cosmos. Advances in Pure Mathematics, 4, 641-648. http://dx.doi.org/10.4236/apm.2014.412073

[117] El Naschie, M.S. (2014) Entanglement of E8E8 Exceptional Lie Symmetry Group Dark Energy, Einstein’s Maximal Total Energy and the Hartle-Hawking No Boundary Proposal as the Explanation for Dark Energy. World Journal of Condensed Matter Physics, 4, 74-77. http://dx.doi.org/10.4236/wjcmp.2014.42011

[118] El Naschie, M.S. (2015) From Fusion Algebra to Cold Fusion or from Pure Reason to Pragmatism. Open Journal of Philosophy, 5, 319-326. http://dx.doi.org/10.4236/ojpp.2015.56040

[119] El Naschie, M.S. (2016) Einstein-Rosen Bridge (ER), Einstein-Podolsky-Rosen Experiment (EPR) and Zero Measure Rindler-KAM Cantorian Spacetime Geometry (ZMG) Are Conceptually Equivalent. Journal of Quantum Information Science, 6, 1-9. http://dx.doi.org/10.4236/jqis.2016.61001

[120] El Naschie, M.S. (2015) A Casimir-Dark Energy Nano Reactor Design—Phase I. Natural Science, 7, 287-298. http://dx.doi.org/10.4236/ns.2015.76032

[121] El Naschie, M.S. (2014) Einstein’s General Relativity and Pure Gravity in a Cosserat and De Sitter-Witten Spacetime Setting as the Explanation of Dark Energy and Cosmic Accelerated Expansion. International Journal of Astronomy and Astrophysics, 4, 332-339. http://dx.doi.org/10.4236/ijaa.2014.42027

[122] El Naschie, M.S. (2015) Application of Dvoretzky’s Theorem of Measure Concentration in Physics and Cosmology. Open Journal of Microphysics, 5, 11-15. http://dx.doi.org/10.4236/ojm.2015.52002

[123] El Naschie, M.S. (2015) Quantum Fractals and the Casimir-Dark Energy Duality-The Road to a Clean Quantum Energy Nano Reactor. Journal of Modern Physics, 6, 1321-1333. http://dx.doi.org/10.4236/jmp.2015.69137

[124] Ho, M.-W., El Naschie, M.S. and Vitello, G. (2015) Is Spacetime Fractal and Quantum Coherent in the Golden Mean? Global Journal of Science Frontier Research, 15, 61-80.

[125] Marek-Crnjac, L. (2015) On El Naschie’s Fractal-Cantorian Space-Time and Dark Energy-A Tutorial Review. Natural Science, 7, 581-598. http://dx.doi.org/10.4236/ns.2015.713058

[126] El Naschie, M.S. (2015) Hubble Scale Dark Energy Meets Nano Scale Casimir Energy and the Rational of Their T-Duality and Mirror Symmetry Equivalence. World Journal of Nano Science and Engineering, 5, 57-67. http://dx.doi.org/10.4236/wjnse.2015.53008

[127] El Naschie, M.S. (2015) The Casimir Effect as a Pure Topological Phenomenon and the Possibility of a Casimir Nano Reactor-A Preliminary Design. American Journal of Nano Research and Application, 3, 33-40.

[128] El Naschie, M.S. (2016) On a Fractal Version of Witten’s M-Theory. International Journal of Astronomy \& Astrophysics, 6, 135-144. http://dx.doi.org/10.4236/ijaa.2016.62011

[129] El Naschie, M.S. (2014) Rindler Space Derivation of Dark Energy. Journal of Modern Physics Applications, 6, 1-10.

[130] El Naschie, M.S. (2015) From Kantian-Reinen Vernunft to the Real Dark Energy Density of the Cosmos via the Measure Concentration of Convex Geometry in Quasi Banach Spacetime. Open Journal of Philosophy, 5, 123-130. http://dx.doi.org/10.4236/ojpp.2015.51014

[131]El Naschie, M.S. (2016) From Witten’s 462 Supercharges of 5-D Branes in Eleven Dimensions to the 95.5 Percent Cosmic Dark Energy Density behind the Accelerated Expansion of the Universe. Journal of Quantum Information Science, 6, 57-61. http://dx.doi.org/10.4236/jqis.2016.62007

[132] El Naschie, M.S. (2016) Quantum Dark Energy from the Hyperbolic Transfinite Cantorian Geometry of the Cosmos. Natural Science, 8, 152-159. http://dx.doi.org/10.4236/ns.2016.83018 
[133] El Naschie, M.S. (2016) On a Quantum Gravity Fractal Spacetime Equation: QRG $\simeq$ HD + FG and Its Application to Dark Energy—Accelerated Cosmic Expansion. Journal of Modern Physics, 7, 729-736. http://dx.doi.org/10.4236/jmp.2016.78069

[134] El Naschie, M.S. (2016) The Emergence of Spacetime from the Quantum in Three Steps. Advances in Pure Mathematics, 6, 446-454. http://dx.doi.org/10.4236/apm.2016.66032

[135] El Naschie, M.S. (2016) Negative Norms in Quantized Strings as Dark Energy Density of the Cosmos. World Journal of Condensed Matter Physics, 6, 63-67. http://dx.doi.org/10.4236/wjcmp.2016.62009

[136] El Naschie, M.S. (2016) On the Stringy Ghosts Which We Call the Missing Dark Energy of the Cosmos. Journal of Applied Mathematics and Physics, 4, 979-987. http://dx.doi.org/10.4236/jamp.2016.46105

[137] El Naschie, M.S. (2016) Einstein’s Dark Energy via Similarity Equivalence, ‘tHooft Dimensional Regularization and Lie Symmetry Groups. International Journal of Astronomy \& Astrophysics, 6, 56-81. http://dx.doi.org/10.4236/ijaa.2016.61005

[138] Ott, E. (1993) Chaos in Dynamical Systems. Cambridge University Press, Cambridge.

[139] Addison, P.S. (1997) Fractals and Chaos: An Illustrated Course. Institute of Physics Publishing, Bristol. http://dx.doi.org/10.1887/0750304006

[140] Ruelle, D. (1991) Chance and Chaos. Princeton University Press, Princeton.

[141] Diacu, F. and Holmes, P. (1996) Celestial Encounters. Princeton University Press, Princeton.

[142] Kapitaniak, T. (1992) Chaotic Oscillators-Theory and Applications. World Scientific, Singapore. http://dx.doi.org/10.1142/9789814360258

[143] El Naschie, M.S. (1998) The Average (n) Sphere Spans a Four Dimensional Manifold. Chaos, Solitons \& Fractals, 9 , 1789-1792. http://dx.doi.org/10.1016/S0960-0779(98)00126-X

[144] El Naschie, M.S. (1998) COBE Satellite Measurement, Hyper Spheres, Superstrings and the Dimension of Spacetime. Chaos, Solitons \& Fractals, 9, 1445-1471. http://dx.doi.org/10.1016/S0960-0779(98)00120-9

[145] He, J.-H. (2005) Transfinite Physics. China Culture \& Science Publishing, Beijing.

[146] El Naschie, M.S. (1998) The Mean Sphere $<$ S > as a Three Manifold in $\mathrm{R}^{4}$. Chaos, Solitons \& Fractals, 9, $1631-1635$. http://dx.doi.org/10.1016/S0960-0779(98)00129-5

[147] Conway, J.H. and Sloane, N.J. (1999) Sphere Packings, Lattices and Groups. Springer, New York. http://dx.doi.org/10.1007/978-1-4757-6568-7

[148] El Naschie, M.S. (2013) Dark Energy from Kaluza-Klein Spacetime and Noether’s Theorem via Lagrangian Multiplier Method. Journal of Modern Physics, 4, 757-760. http://dx.doi.org/10.4236/jmp.2013.46103

[149] El Naschie, M.S. (2008) Kaluza-Klein Unification—Some Possible Extensions. Chaos, Solitons \& Fractals, 37, 16-22. http://dx.doi.org/10.1016/j.chaos.2007.09.079

[150] El Naschie, M.S. (2013) Electromagnetic and Gravitational Origin of Dark Energy in Kaluza-Klein D = 5 Spacetime. Piers Proceedings, Stockholm, 12-15 August 2013, 94-97.

[151] Polchinski, J. (1998) String Theory Vol. 1. Cambridge University Press, Cambridge. (See in Particular p. 22, Equation (1.3.33))

[152] El Naschie, M.S., Olsen, S., He, J.H., Nada, S., Marek-Crnjac, L. and Helal, A. (2012) On the Need for Fractal Logic in High Energy Quantum Physics. International Journal of Modern Nonlinear Theory and Application, 1, 84-92. http://dx.doi.org/10.4236/ijmnta.2012.13012

[153] El Naschie, M.S. (1991) Physics-Like Mathematics of Chaos and Ergodicity in Four Dimensions. Proceedings of the 13th World Congress on Computation and Applied Mathematics, 2, 851-852.

[154] El Naschie, M.S. (1998) Von Neumann Geometry and E-Infinity Quantum Spacetime. Chaos, Solitons \& Fractals, 9, 2023-2030.

[155] El Naschie, M.S. (2004) Small World Network, E-Infinity Topology and the Mass Spectrum of High Energy Particle Physics. Chaos, Solitons \& Fractals, 19, 689-697. http://dx.doi.org/10.1016/S0960-0779(03)00337-0 


\section{Submit or recommend next manuscript to SCIRP and we will provide best service for you:}

Accepting pre-submission inquiries through Email, Facebook, Linkedin, Twitter, etc A wide selection of journals (inclusive of 9 subjects, more than 200 journals)

Providing a 24-hour high-quality service

User-friendly online submission system

Fair and swift peer-review system

Efficient typesetting and proofreading procedure

Display of the result of downloads and visits, as well as the number of cited articles

Maximum dissemination of your research work

Submit your manuscript at: http://papersubmission.scirp.org/ 